\title{
Order-by-Disorder Degeneracy Lifting of Interacting Bosons on the Dice Lattice
}

\author{
Matjaž Payrits and Ryan Barnett \\ Department of Mathematics, Imperial College London, London SW7 2AZ, United Kingdom
}

\begin{abstract}
Motivated by recent experimental progress in the realization of synthetic gauge fields in systems of ultracold atoms, we consider interacting bosons on the dice lattice with half flux per plaquette. All bands of the non-interacting spectrum of this system were previously found to have the remarkable property of being completely dispersionless. We show that degeneracies remain when interactions are treated at the level of mean field theory, and the ground state exhibits vortex lattice configurations already established in the simpler XY model in the same geometry. We argue that including quantum and thermal fluctuations will select a unique vortex lattice up to overall symmetries based on the order-by-disorder mechanism. We verify the stability of the selected state by analyzing the condensate depletion. The latter is shown to exhibit an unusual non-monotonic behavior as a function of the interaction parameters which can be understood as a consequence of the dispersionless nature of the non-interacting spectrum. Finally, we comment on the role of domain walls which have interactions mediated through fluctuations.
\end{abstract}

PACS numbers: 03.75.Hh, 67.85.Hj, 74.81.Fa

\section{INTRODUCTION}

The experimental realization of synthetic gauge fields in systems of ultracold atoms by several groups has reinvigorated interest in lattice boson systems under large effective magnetic fields (for a recent review, see Ref. [1]). Recently, the Hofstadter model, which describes particles on a square lattice under an external effective magnetic field, was realized in a regime where the magnetic length is on the order of the lattice constant [2, 3]. For electrons in solid state materials, such field strengths would correspond to extremely large magnetic fields on the order of $10^{4}$ Tesla. Additionally, more complex optical lattices can be realized, including the Kagomé lattice [4, which opens the door to exploring the generalizations of the Hofstadter model to non-Bravais lattices.

Weakly interacting bosons at low temperature on a lattice under an external effective magnetic field, created from rotation or a synthetic gauge field, will generally form a vortex lattice [5, 6. When the magnetic length is large compared to the lattice constant, the familiar triangular Abrikosov vortex lattice is formed. However, when the magnetic length is on the order of the lattice constant, there can be a subtle interplay between these two length scales and a variety of non-triangular lattices can be formed as seen from the minimization of the XY model or the Gross-Pitaevskii energy functional [7-10]. Such mean field theories are typically sufficient to determine the ground state of these systems up to overall symmetries.

The dice lattice under an effective magnetic field is an exception to this paradigm. For the particular case of half a flux quantum per plaquette, it is known that its single-particle spectrum in the tight-binding approximation exhibits three completely flat bands [1]. Interactions are therefore necessarily of crucial importance in determining the possible phases of this system. Flat band systems with nonzero Chern numbers have also been proposed as candidates for realizing fractional Chern insu- lators 1214 . Though the Chern numbers of the bands in the present analysis of the dice lattice vanish, it was found in Ref. [15] that it is possible to obtain bands with non-zero Chern number through the introduction of spin-orbit coupling (though this introduces dispersion to the bands). Additionally, flat band systems have received considerable attention within the context of ultracold gases [4, 16 21]. An analysis by Korshunov of the related XY model on the dice lattice with half flux per plaquette shows that such degeneracies persist in the vortex lattice structures 22 24]. In particular, it was found that the four periodic vortex lattice structures shown in Fig. 2 are all degenerate ground states of the XY model.

The dice lattice first garnered considerable attention in the context of the so-called topological or AharonovBohm localization mechanism [11, 25, 26]. Later studies have explored the phase diagram of the Bose-Hubbard model in a dice geometry in various approximations [27, 28, and shown that it can give rise to effective DiracWeyl fermions 29. In Ref. 30] it was shown that an order-by-disorder mechanism in the dice lattice BoseHubbard model yields a Vortex-Peierls state near the Mott insulating - superfluid transition. Most recently, ground state quantum phases of the model were determined in the lowest Landau level regime where it is appropriate to project into the lowest single-particle band of the system 31. Some of the exotic properties associated with the single-particle spectrum of the dice lattice have also been observed experimentally 32,34 .

In this work, we consider interacting bosons with nearest-neighbor hopping on the dice lattice with half flux per plaquette, described by the Bose-Hubbard model. We show that the same periodic vortex lattices as established for the XY model 23] are degenerate energetic minima at the level of Gross Pitaevskii mean field theory. As these degeneracies are not protected by any symmetry, they are expected to be lifted by the order-by-disorder mechanism [35, 36]. Although quantum order-by-disorder is perhaps most familiar from 
frustrated magnetism, it can also play important roles in ultracold atomic gases [19, 37, 40]. We in particular show that quantum and thermal fluctuations at quadratic order completely lift the degeneracy between the four candidate vortex lattices shown in Fig. 2, where state (b) acquires the lowest energy. In contrast, it was found that thermal fluctuations in the analogous classical XY model on the dice lattice at quadratic order do not lift the degeneracy between the ground state vortex configurations and one must rely on anharmonic fluctuations which are estimated to be small on experimental scales 24. It should be noted that Ref. 30] performed a similar analysis near the Mott insulator-superfluid transition, whereas we focus on the deep superfluid regime.

This paper is organized as follows. In Sec. II we set up the theoretical problem of bosons hopping on the dice lattice under an effective magnetic field. For completeness, we also review the single-particle spectrum of flat bands originally found in Ref. 11. In Sec. III we determine the periodic mean field vortex lattice configurations. These are shown to have the same phase structure as reported on studies of the XY model [22]. Unlike the XY model, the GP energy functional has local density degrees of freedom, which are shown to take on two distinct values representing the two inequivalent sites in the dice lattice. In Sec. IV we describe the computation of the collective excitations about each of the four periodic vortex configurations. We will show that harmonic fluctuations completely lift the degeneracy between the configurations through the order-by-disorder mechanism. We address the stability of the proposed state with respect to quantum and thermal depletion. As is common in twodimensional systems [41, the thermal depletion exhibits a logarithmic infrared divergence which is removed for finite-sized systems. We show that the depletion is small for realistic experimental parameters. Interestingly, the depletion shows a non-monotonic behavior as a function of the interaction parameters which can be attributed to the flat band structure. Finally, in Sec. V] the results are discussed and the work is concluded.

\section{THEORETICAL SETUP}

We consider bosons in the dice lattice potential, also referred to as the $\tau_{3}$ lattice [1], shown in Fig. 1. The bosons are treated within the tight-binding approximation with nearest-neighbor hopping. Experimental proposals to engineer the dice optical lattice under an effective magnetic field are provided in [28 31. Josephson junction arrays with the appropriate geometry provide another physically feasible avenue of realizing the dice lattice experimentally.

We label the unit cells of the lattice with an integer $n$ and denote the corresponding unit cell location by $\boldsymbol{R}_{n}$. When the unit-cell/basis-vector decomposition is important, we label the site displaced from the origin of the $n$-th unit cell by the basis vector $\mathbf{b}_{\gamma}$ with the pair $(n, \gamma)$.
Otherwise we label each site by a single integer $i$ and denote its location by $\boldsymbol{r}_{i}$. We consider the system subjected to a synthetic gauge field with vector potential $\boldsymbol{A}$ such that its line integral around any lattice plaquette equals $\pi$. We can draw clear analogies with electromagnetism: by considering the example of a particle of charge $q$ in the presence of an electromagnetic potential $\boldsymbol{A}_{\mathrm{EM}}, \boldsymbol{A}$ is found to be analogous to $\frac{2 \pi}{\Phi_{0}} \boldsymbol{A}_{\mathrm{EM}}$ where $\Phi_{0}=h / q$ is the charge- $q$ elementary magnetic flux quantum. Likewise, $\int_{\mathcal{C}} \boldsymbol{A} \cdot \mathrm{d} \boldsymbol{r}$, with $\mathcal{C}$ a cyclic path along the edges of a plaquette, is analogous to the charged particle's corresponding Aharonov-Bohm phase. Since the latter equals $\pi$ when the plaquette is threaded by half an elementary magnetic flux, our synthetic gauge field configuration is analogously referred to as half(-elementary)flux-per-plaquette.

In the continuum, electromagnetism is introduced into the Hamiltonian via minimal coupling, i.e. $\hat{\boldsymbol{p}} \rightarrow \hat{\boldsymbol{p}}-q \hat{\boldsymbol{A}}$. The tight-binding equivalent of this procedure is Peierls substitution, i.e. substituting pairs of creation and annihilation operators according to $\hat{a}_{i}^{\dagger} \hat{a}_{j} \rightarrow \mathrm{e}^{\mathrm{i} A_{i j}} \hat{a}_{i}^{\dagger} \hat{a}_{j}$, where $\hat{a}_{i}$ is the annihilation operator for the $i$-th site and $A_{i j}=\int_{\boldsymbol{r}_{j}}^{\boldsymbol{r}_{i}} \boldsymbol{A}(\boldsymbol{r}) \cdot \mathrm{d} \boldsymbol{r}$ is the line integral of the vector potential between sites $j$ and $i$ involved in the hopping. The noninteracting part of the Hamiltonian is thus $\hat{H}_{0}=-t \sum_{\langle i j\rangle}\left(\mathrm{e}^{\mathrm{i} A_{i j}} \hat{a}_{i}^{\dagger} \hat{a}_{j}+\right.$ h.c. $)$ where the sum is taken over all pairs of nearest neighbor sites.

We assume the inter-site interactions are negligibly weak. The interaction part of the Hamiltonian thus consists of terms of the form $\frac{1}{2} U_{i} \hat{a}_{i}^{\dagger} \hat{a}_{i}^{\dagger} \hat{a}_{i} \hat{a}_{i}$, where $U_{i}$ is the positive onsite interaction. It is evident from Fig. 1 that there are two qualitatively different types of sites with coordination numbers 6 and 3. Following Ref. [25], we refer to these as $h u b\left(^{*}\right)$ sites and rim $(\Delta)$ sites, respectively. We accordingly regard $U_{*}$ and $U_{\Delta}$ as independent parameters. Introducing the chemical potential $\mu$, the full Bose-Hubbard model on the dice lattice reads

$$
\begin{aligned}
\hat{H}=-t \sum_{\langle i j\rangle} & \left(\mathrm{e}^{\mathrm{i} A_{i j}} \hat{a}_{i}^{\dagger} \hat{a}_{j}+\text { H.c. }\right) \\
& +\sum_{i}\left(\frac{1}{2} U_{i} \hat{a}_{i}^{\dagger} \hat{a}_{i}^{\dagger} \hat{a}_{i} \hat{a}_{i}-\mu \hat{a}_{i}^{\dagger} \hat{a}_{i}\right) .
\end{aligned}
$$

\section{A. Single-particle spectrum}

For completeness, we review the single particle spectrum of the dice lattice at half-flux per plaquette which was first derived in Ref. [11. At the non-interacting level this system already displays some remarkable features. To calculate the spectrum we use the momentum space creation and annihilation operators defined as $\hat{a}_{\boldsymbol{k} \gamma}=\frac{1}{\sqrt{N}} \sum_{n} \hat{a}_{n \gamma} \mathrm{e}^{-\mathrm{i} \boldsymbol{k} \cdot \boldsymbol{R}_{n}}$ where $N$ is the number of unit cells in the system. We adopt the convenient gauge of Ref. 31, shown in Fig. 1. where the effective hopping 


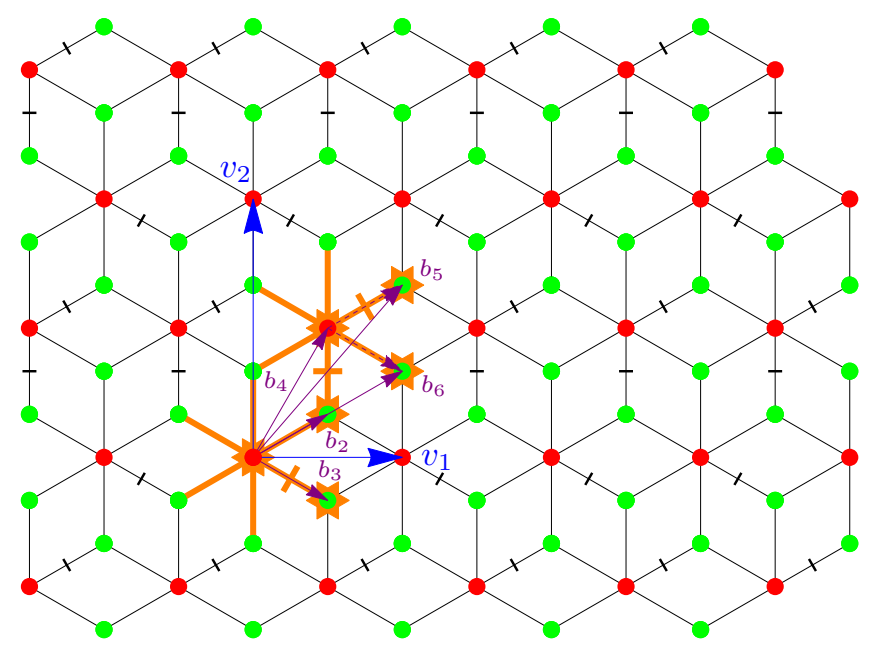

FIG. 1. (Color online) The dice lattice under an effective magnetic field using the gauge of Ref. 31. There are two types of sites: hub sites with a coordination number of 6 and rim sites with a coordination number of 3 . The links and sites outlined in orange comprise the half-flux-per-plaquette magnetic unit cell. Particles acquire a phase of $\pi$ when hopping across crossed links and no phase when hopping across uncrossed links. The lattice vectors $\boldsymbol{v}_{1}$ and $\boldsymbol{v}_{2}$ can be chosen to be orthogonal, as in the figure where $\boldsymbol{v}_{1}=(1,0)$, $\boldsymbol{v}_{2}=(0, \sqrt{3})$. For convenience we have set the lattice constant to unity. The lattice can thus be viewed as a rectangular Bravais lattice with a 6 -fold basis. The basis vectors are $\boldsymbol{b}_{1}=\mathbf{0}, \boldsymbol{b}_{2}=(1 / 2,1 / 2 \sqrt{3}), \boldsymbol{b}_{3}=(1 / 2,-1 / 2 \sqrt{3}), \boldsymbol{b}_{4}=$ $(1 / 2, \sqrt{3} / 2), \boldsymbol{b}_{5}=\boldsymbol{b}_{2}+\boldsymbol{b}_{4}$ and $\boldsymbol{b}_{6}=\boldsymbol{b}_{3}+\boldsymbol{b}_{4}$. In the absence of a gauge field the smallest unit cell is half the size and we can take $\boldsymbol{v}_{1}$ and $\boldsymbol{b}_{4}$ for the lattice vectors and $\boldsymbol{b}_{1-3}$ for the three basis vectors.

parameters are real. Owing to the periodicity of the lattice we can rewrite the non-interacting portion of the Hamiltonian in Eq. (1) as

$$
\hat{H}_{0}=\sum_{\boldsymbol{k}} \hat{\boldsymbol{a}}_{\boldsymbol{k}}^{\dagger} H_{0}(\boldsymbol{k}) \hat{\boldsymbol{a}}_{\boldsymbol{k}}
$$

where $\hat{\boldsymbol{a}}_{\boldsymbol{k}}=\left[\hat{a}_{\boldsymbol{k} 1}, \hat{a}_{\boldsymbol{k} 2}, \cdots, \hat{a}_{\boldsymbol{k} 6}\right]^{\top}, H_{0}(\boldsymbol{k})$ is a 6 -by-6 Hermitian matrix, and the summation is over the first Brillouin zone. By inserting a full set of eigenvectors $\sum_{\gamma=1}^{6} \boldsymbol{u}_{\boldsymbol{k} \gamma} \boldsymbol{u}_{\boldsymbol{k} \gamma}^{\dagger}$ on both sides of $H_{0}(\boldsymbol{k})$ in Eq. (2) we can express the Hamiltonian in terms of new quasiparticle operators $\hat{\alpha}_{\boldsymbol{k} \gamma}=\boldsymbol{u}_{\boldsymbol{k} \gamma}^{\dagger} \hat{\boldsymbol{a}}_{\boldsymbol{k}}$ and their corresponding eigenvalues $\lambda_{\boldsymbol{k} \gamma}$ as

$$
\hat{H}_{0}=\sum_{\boldsymbol{k} \gamma} \lambda_{\boldsymbol{k} \gamma} \hat{\alpha}_{\boldsymbol{k} \gamma}^{\dagger} \hat{\alpha}_{\boldsymbol{k} \gamma}
$$

The fascinating outcome is that the energies $\lambda_{\boldsymbol{k} \gamma}$ have no dispersion and remain constant throughout the Brillouin zone. There are three doubly degenerate bands with $\lambda_{\boldsymbol{k} \gamma}= \pm \sqrt{6} t, 0$. For the lowest and highest bands this follows from the fact that their states can be expressed as a sum of completely localized eigenstates.
The Wannier functions, obtained from the Fourier transform of these extended Bloch wave functions, provide a particularly convenient basis for describing the single-particle states. For the highest and lowest energy bands they are both eigenstates of the non-interacting Hamiltonian and completely localized. For both of these bands they span a hub site and its six surrounding rim sites. The amplitude on the hub is $1 / \sqrt{2}$ and $1 / \sqrt{12}$ on the rim sites. The phase of rim site $j$ relative to the central hub $i$ is, in the gauge of Fig. 1. simply $A_{j i}$ in the lowest band and $\pi-A_{j i}$ in the top band, i.e. either 0 or $\pi$ in both cases. The existence of these localized states does not fall under any of the disorder-based localization paradigms, such as Anderson localization [42], but follows solely from destructive interference within the so-called Aharonov-Bohm cages on the lattice [11. The Wannier functions corresponding to the zero-energy eigenstates, on the other hand, are only exponentially localized, so this simple explanation of flatness is not applicable for this case.

\section{MEAN FIELD THEORY}

We proceed by reintroducing interactions and finding the ground states at the mean-field level by solving the Gross-Pitaevskii equation. This is equivalent to assuming that the wave function can be written as a tensor product of independent coherent states for each site. Accordingly, we can replace operators with c-numbers

$$
\hat{a}_{i} \rightarrow a_{i}=\sqrt{n_{i}} \mathrm{e}^{\mathrm{i} \theta_{i}}
$$

with similar expressions for momentum-space quantities. Here $n$ and $\theta$ are the density and phase variables. We will later be able to find elementary excitations about these states by means of Bogoliubov theory.

Given the simple structure of the single-particle spectrum, it is reasonable to ask whether there exist any states that simultaneously minimize both the singleparticle and the interaction part of the mean-field energy. The former is true when the state can be constructed as a linear combination of states in the lowest single-particle band and the latter when it gives rise to uniform densities $n_{*}$ and $n_{\Delta}$ on the hub and rim sublattices, respectively. By writing the state as a linear combination of lowestband eigenstates, one finds that such uniform densities can only be obtained when $U_{\Delta} / U_{*}=2$. For future reference we term this parameter configuration the special point. Besides uniform densities, the state also has a simple phase picture. In particular, we only encounter three distinct magnitudes of gauge invariant phase differences. These are defined as

$$
\Phi_{i j}=\theta_{i}-\theta_{j}-A_{i j}
$$

and are indeed independent of our chosen gauge. We derive their values in the next section.

We conjecture that the states globally minimizing the total mean field energy away from the special point retain 
uniform densities on both sublattices. This is motivated by the fact that the proposed states merge with what are provably the only global minima at the special point and by our failing to find a physically reasonable mechanism capable of breaking the density symmetry. In the following section we show that the necessary condition of the states remaining local energy minima is satisfied. At the uniform sublattice density configurations we can furthermore follow 24 to show that the phase profiles minimizing the energy are identical to those at the special point.

\section{A. Mean field calculation of sublattice densities}

The mean field energy of the Hamiltonian (1) is

$$
E=-2 t \sum_{\langle i j\rangle} \sqrt{n_{i} n_{j}} \cos \left(\Phi_{i j}\right)+\frac{1}{2} \sum_{i} U_{i} n_{i}^{2}-\mu \sum_{i} n_{i}
$$

We will derive the equations of motion with the corresponding Lagrangian

$$
L=\sum_{i}\left(-n_{i} \dot{\theta}_{i}\right)-E .
$$

Expressed in terms of gauge invariant quantities, the Euler-Lagrange equations read

$$
\begin{aligned}
\dot{n}_{i} & =2 t \sum_{j \in \mathcal{N}_{i}} \sqrt{n_{i} n_{j}} \sin \Phi_{i j} \\
\dot{\Phi}_{i j} & =t \sum_{i^{\prime} \in \mathcal{N}_{i}} \sqrt{\frac{n_{i^{\prime}}}{n_{i}}} \cos \Phi_{i i^{\prime}}-t \sum_{j^{\prime} \in \mathcal{N}_{j}} \sqrt{\frac{n_{j^{\prime}}}{n_{j}}} \cos \Phi_{j j^{\prime}} \\
& +U_{j} n_{j}-U_{i} n_{i} .
\end{aligned}
$$

In this expression, $\mathcal{N}_{i}$ denotes the set of all sites neighboring site $i$. For the ground state we demand that the time derivatives on the LHS be zero.

We now insert the key assumption of uniform sublattice densities. Taking into account the overall geometry, the second equation yields

$U_{*} n_{*}-U_{\Delta} n_{\Delta}=t \sqrt{\frac{n_{\Delta}}{n_{*}}} \sum_{i^{\prime} \in \mathcal{N}_{*}} \cos \Phi_{* i^{\prime}}-t \sqrt{\frac{n_{*}}{n_{\Delta}}} \sum_{j^{\prime} \in \mathcal{N}_{\Delta}} \cos \Phi_{\Delta j}$.

As remarked before, the phase profiles occurring at the special point still solve the equations. Let us denote the three distinct phase difference magnitudes comprising them by $\Phi_{l}>\Phi_{m}>\Phi_{s}>0(l, m, s$ for large, medium, small). Since the factor $\sqrt{n_{i} n_{j}}$ equals $\sqrt{n_{\Delta} n_{*}}$ for any neighboring $i$ and $j$, we can rewrite equation (8), the continuity equation, as $\sin \Phi_{l}=\sin \Phi_{m}+\sin \Phi_{s}$. The condition that the sum of phase differences around a plaquette equal $\pm \pi$ imposes the restrictions $2 \Phi_{s}+2 \Phi_{l}=\pi$ and $-\Phi_{s}+2 \Phi_{m}+\Phi_{l}=\pi$. This system of equations yields $\Phi_{s} \approx 9.74^{\circ}, \Phi_{m} \approx 54.74^{\circ}$ and $\Phi_{l} \approx 80.26^{\circ}$, along with the useful identity

$$
\mathrm{e}^{\mathrm{i} \Phi_{s}}+\mathrm{e}^{\mathrm{i} \Phi_{m}}+\mathrm{e}^{-\mathrm{i} \Phi_{l}}=\sqrt{3} .
$$

While it can easily be seen that each of these three phase differences appears exactly once for links surrounding any rim site, it can also be shown that each appears exactly twice among the links surrounding any hub site, though the procedure is tedious.

This phase configuration is identical to the one obtained by Korshunov for the dice lattice XY model [22]. This is so because the form of Eq. (8) is the same in both cases, as it does not depend on the local interaction terms of the Hamiltonian. Furthermore, the factor $\sqrt{n_{i} n_{j}}$ in Eq. 8 is constant for all pairs of neighboring sites in both cases. It can thus be factored out when considering the ground state. In Ref. 22] this is due to the author's explicitly taking a uniform density across all sites, while in our case it is due to the alternating nature of the uniform density hub and rim sublattices.

We can in fact easily determine the sublattice density values. Taking the features of the phase configuration and Eq. 111 into account, Eq. 10 simplifies to

$$
U_{*} n_{*}-U_{\Delta} n_{\Delta}=\sqrt{3} t \frac{2 n_{\Delta}-n_{*}}{\sqrt{n_{\Delta} n_{*}}} .
$$

Given the two interaction strengths, this equation can be solved to determine the ratio of densities on the hub and rim sites, $n_{*} / n_{\Delta}$. Note that at the special point, where $U_{\Delta}=2 U_{*}$, one has the simplest case $n_{*}=2 n_{\Delta}$, as expected. Finally, with this solution the chemical potential is found to be

$$
\mu=U_{*} n_{*}-2 t \sqrt{\frac{3 n_{\Delta}}{n_{*}}}=U_{\Delta} n_{\Delta}-t \sqrt{\frac{3 n_{*}}{n_{\Delta}}}
$$

\section{B. Mean field periodic ground states}

We can assign to each plaquette a vorticity of either $\pi$ or $-\pi$. Through a qualitative comparison of this vortex lattice with the two-dimensional Coulomb gas, relevant since neutral superfluid vortices are known to have approximately a logarithmic interaction, one can argue that the most energetically favorable configuration will have each vortex surrounded by as many neighbors of the opposite vorticity as possible. The vortices are pinned to the sites of the dual lattice which in this case is the Kagomé lattice. The geometric frustration of the Kagomé lattice prevents the possibility of a purely local prescription for the distribution of vortices minimizing the energy. The vortex configuration of mean field ground states is demonstrably composed of chains of likevortices of length three 43 .

Perhaps the simplest such state is shown in Fig. 2(a). We can obtain all other applicable states with only the three gauge invariant phase differences introduced above by rearranging the phase differences along a variety of infinite sequences of plaquettes in which every pair of neighboring plaquettes shares just a single vertex. We can think of this process as the insertion of two types of zero-energy domain walls into state (a). We will refer 


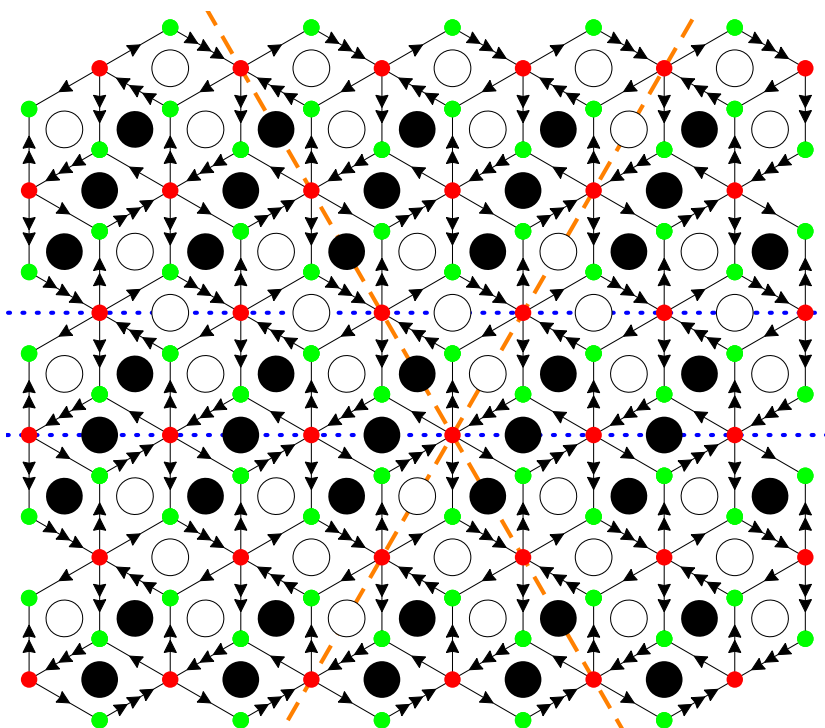

(a)

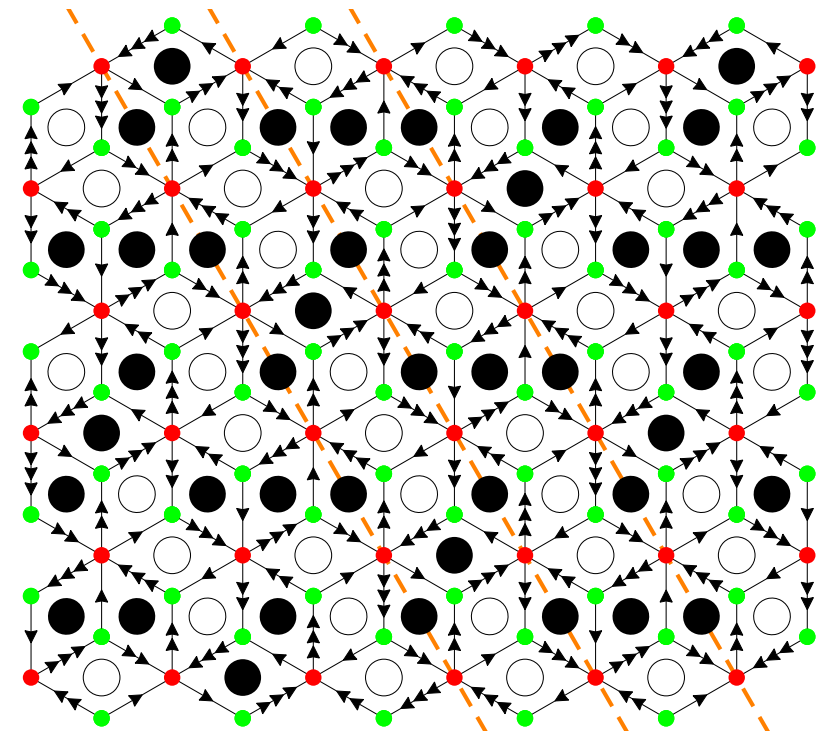

(b)

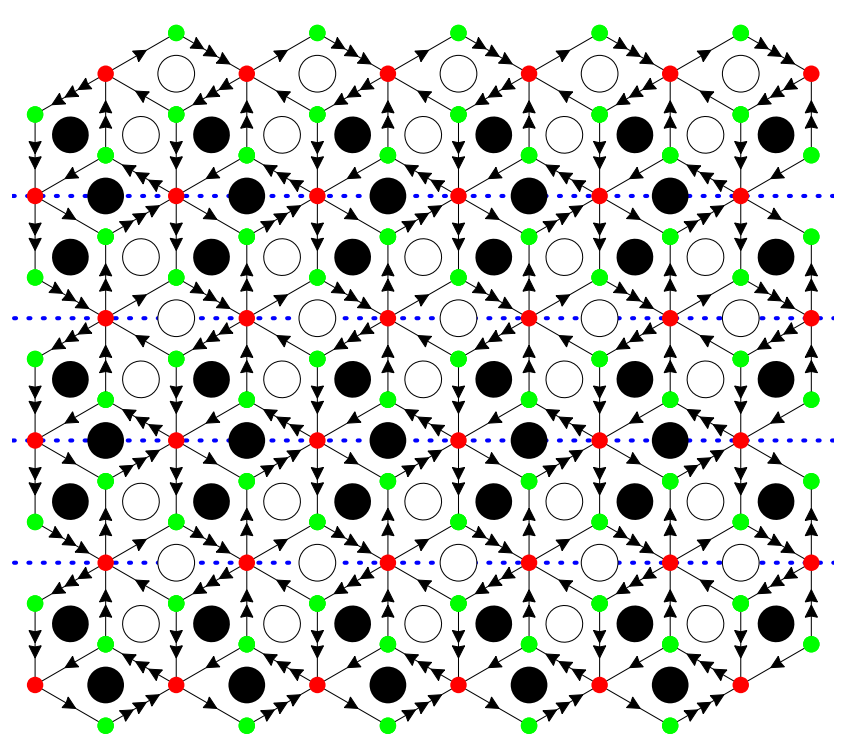

(c)

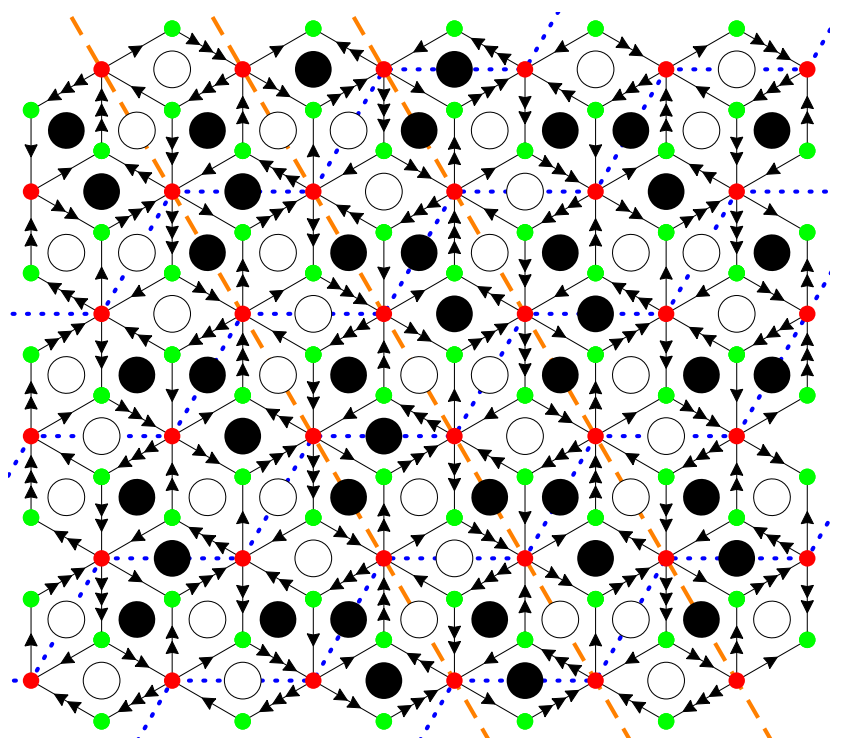

(d)

FIG. 2. (Color online) The four small unit cell periodic mean field ground states. The single, double and triple arrows represent gauge invariant phase differences $\Phi_{s}, \Phi_{m}$ and $\Phi_{l}$ across links, respectively, and the black (white) disks represent positive (negative) plaquette vorticities. The dashed and dotted lines signify locations of possible domain wall insertions (figure (a)) or domain walls themselves (all other figures). The dashed orange lines represent type I domain walls while the blue dotted lines represent type II domain walls.

to the domain walls we can insert parallel to the dashed lines in Fig. 2(a) as type I domain walls 44 and the ones we can insert parallel to the dotted lines as type II domain walls. Inserting a type I domain wall splits the lattice into two regions with orientations of the vortex triads not parallel to the wall differing by $60^{\circ}$. A type II domain wall bends the triads it crosses and establishes a mirror symmetry between both of its sides. Type II domain walls also bend by $60^{\circ}$ whenever they cross a type I domain wall [45.
The unit cell of vortex state (a) contains six lattice sites. It is twelvefold degenerate under the following geometric transformations that preserve the Hamiltonian but not the state: translations by $\pm \boldsymbol{b}_{4}$ or $\boldsymbol{b}_{4}-\boldsymbol{b}_{1}$, using the notation of Fig. 1, contributing a factor 2 to the geometric degeneracy, the combination of time (arrow) reversal and spatial inversion, contributing another factor of 2 , and $\pm 2 \pi / 3$ rotations about any site, contributing the final factor of 3. By inserting all possible type II domain walls into (a) we obtain another twelvefold degen- 
erate state with six sites per unit cell, shown in Fig. 2(c), not related to state (a) by geometric symmetries. Inserting all possible type I domain walls into state (a) similarly yields the state shown in Fig. 2(b) with twelve sites per unit cell. Further inserting all possible type II domain walls into (b) yields the state shown in Fig. 2(d), also containing twelve sites per unit cell. States (b) and (d) have a fourfold translational degeneracy, so their total geometric degeneracy is 24-fold. Taking geometric multiplicities into account this yields a total of 72 small unit cell mean field periodic states, or SMPS's. All other uniform sublattice periodic mean field ground states can be obtained by gluing together the unit cells of the above four classes of SMPS's 24].

It should be noted that given two asymptotically domain wall-free regions, such that, for instance, the vortex lattice is one of the 72 SMPS's on the far left and a distinct SMPS on the far right, it is not in general possible to consistently interpolate between the two through a sequence of SMPS regions, i.e. state (a-d)-like regions, glued by zero-energy domain walls. This implies either the possibility of massive, i.e. energetically costly, domain walls and point defects, or global instabilities of such asymptotic configurations. We were unable to obtain concrete results regarding this issue, but include a brief speculative discussion of possible scenarios in the conclusion.

The geometric degeneracies discussed above originate from true symmetries of the Hamiltonian and are as such not expected to be lifted by fluctuations. In the following we focus on the effects of quantum zero-point and thermal fluctuations on the four classes of accidentally degenerate periodic mean field states (a-d) of Fig. 2, Later we also briefly comment on the expected role of domain wall interactions in more general states.

\section{ORDER-BY-DISORDER DEGENERACY LIFTING}

Through the mechanism of order-by-disorder 35, quantum and thermal fluctuations act to remove accidental mean-field degeneracies, i.e. degeneracies not protected by symmetries of the Hamiltonian. In quantitative terms, the state whose fluctuations yield the lowest Helmholtz free energy $F=-\frac{1}{\beta} \ln Z$, where $Z=$ $\operatorname{Tr}\left(e^{-\beta \hat{H}}\right)$ is the partition function and $\beta=1 / k_{B} T$, is selected. At the level of Bogoliubov theory, the excitation spectrum is described by independent harmonic oscillators, and so we have

$$
Z=\sum_{n_{i}} \mathrm{e}^{-\beta \hbar \sum_{j} \omega_{j}\left(n_{j}+\frac{1}{2}\right)}=\prod_{j} \mathrm{e}^{-\frac{\beta E_{j}}{2}} \frac{1}{1-\mathrm{e}^{-\beta E_{j}}}
$$

From this the free energy can be found to be

$$
F=\frac{1}{2} \sum_{j} E_{j}+\beta^{-1} \sum_{j} \ln \left(1-\mathrm{e}^{-\beta E_{j}}\right) .
$$

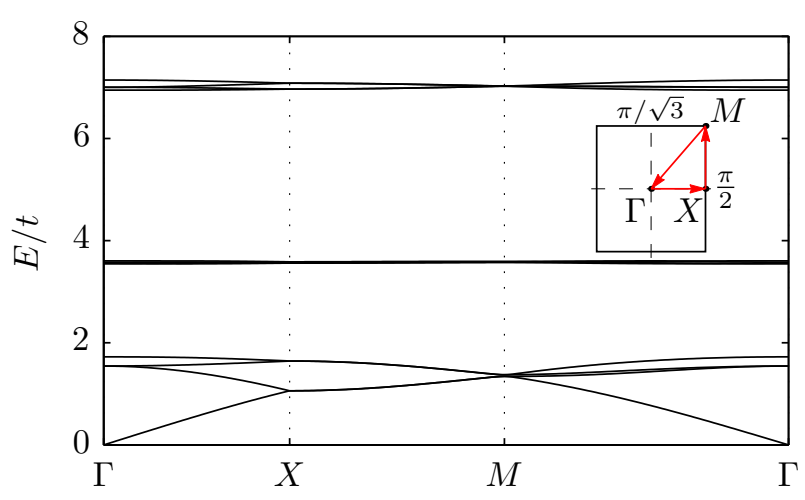

FIG. 3. The twelve Bogoliubov modes about ground state (b) from Fig. 2 at $U_{*}=U_{\Delta}, U_{*} / t=.5$ and $n_{*}=6$. As the interaction strengths $U_{*, \Delta}$ decrease, the bands flatten and the gaps between them approach $\sqrt{6} t$. At $U_{*, \Delta}=0$ we recover the dispersionless degenerate single-particle spectrum.

The first term corresponds to the zero-point quantum contribution to the free energy and the second term to the contribution of thermal fluctuations. In our case the index $j$ in Eq. (15) is a label for momentum and band index.

\section{A. Collective Excitation Spectrum}

We now derive the collective excitation spectrum of Eq. (1) at the level of Bogoliubov theory. This involves expressing the annihilation operators as $\hat{a}_{i}=a_{i}+\delta \hat{a}_{i}$, where $a_{i}$ are the mean-field c-values from Eq. (4). We expand the full hamiltonian in $\delta \hat{a}_{i}$, keeping terms up to quadratic order. The first order term always vanishes as we are expanding about a minimum while the zeroth order term gives the degenerate mean-field energies. Thus we focus on the second order contribution.

Substituting $\hat{a}_{i}=a_{i}+\delta \hat{a}_{i}$ into Eq. (1), and using the chemical potential given in Eq. (13), one finds the quadratic Hamiltonian

$$
\begin{aligned}
\delta \hat{H} & =-t \sum_{\langle i j\rangle}\left(\mathrm{e}^{\mathrm{i} A_{i j}} \delta \hat{a}_{i}^{\dagger} \delta \hat{a}_{j}+\text { H.c. }\right)+\sum_{i}\left(U_{i} n_{i}+G_{i}\right) \delta \hat{a}_{i}^{\dagger} \delta \hat{a}_{i} \\
& +\sum_{i} \frac{U_{i}}{2}\left(a_{i}^{*} a_{i}^{*} \delta \hat{a}_{i} \delta \hat{a}_{i}+a_{i} a_{i} \delta \hat{a}_{i}^{\dagger} \delta \hat{a}_{i}^{\dagger}\right)
\end{aligned}
$$

where $n_{i}=\left|a_{i}\right|^{2}, G_{*}=2 t \sqrt{\frac{3 n_{\Delta}}{n_{*}}}$, and $G_{\Delta}=t \sqrt{\frac{3 n_{*}}{n_{\Delta}}}$. It greatly simplifies the analysis to perform the gauge transformation $\delta \hat{a}_{i} \rightarrow e^{i \theta_{i}} \delta \hat{a}_{i}$ at this stage. This results in the following gauge invariant Bogoliubov Hamiltonian

$$
\begin{aligned}
\hat{H}_{B} & =-t \sum_{\langle i j\rangle}\left(\mathrm{e}^{-\mathrm{i} \Phi_{i j}} \delta \hat{a}_{i}^{\dagger} \delta \hat{a}_{j}+\text { H.c. }\right) \\
& +\sum_{i}\left[\left(U_{i} n_{i}+G_{i}\right) \delta \hat{a}_{i}^{\dagger} \delta \hat{a}_{i}+\frac{U_{i} n_{i}}{2}\left(\delta \hat{a}_{i} \delta \hat{a}_{i}+\delta \hat{a}_{i}^{\dagger} \delta \hat{a}_{i}^{\dagger}\right)\right] .
\end{aligned}
$$


The gauge invariant phase differences $\Phi_{i j}$ here are precisely those introduced in Sec. III A.

We can again define momentum space operators $\delta \hat{a}_{\boldsymbol{k} \gamma}=\frac{1}{\sqrt{N}} \sum_{n} \delta \hat{a}_{n \gamma} \mathrm{e}^{-\mathrm{i} \boldsymbol{k} \cdot \boldsymbol{R}_{n}}$ with $\gamma=1, \cdots, M$, where $M$ is the number of sites per unit cell. By expressing the Hamiltonian in terms of these operators we obtain, up to a constant energy shift (equal for all mean-field states), the Hamiltonian in the form

$$
\hat{H}_{B}=\sum_{\boldsymbol{k}} \boldsymbol{\delta} \hat{\boldsymbol{a}}_{\boldsymbol{k}}^{\dagger} H_{B}(\boldsymbol{k}) \boldsymbol{\delta} \hat{\boldsymbol{a}}_{\boldsymbol{k}}
$$

where $\boldsymbol{\delta} \hat{\boldsymbol{a}}_{\boldsymbol{k}}=\left[\delta \hat{a}_{\boldsymbol{k} 1}, \cdots, \delta \hat{a}_{\boldsymbol{k} M}, \delta \hat{a}_{-\boldsymbol{k} 1}^{\dagger}, \cdots, \delta \hat{a}_{-\boldsymbol{k} M}^{\dagger}\right]^{\top}$ and

$$
H_{B}(\boldsymbol{k})=\left[\begin{array}{c|c}
C_{\boldsymbol{k}} & D \\
\hline D & C_{-\boldsymbol{k}}^{\top}
\end{array}\right] .
$$

Here $D$ is a diagonal matrix with $U_{*} n_{*}\left(U_{\Delta} n_{\Delta}\right)$ entries for hub (rim) sites. $C_{\boldsymbol{k}}=H_{0}(\boldsymbol{k})+G+D$, where $H_{0}(\boldsymbol{k})$ is the single-particle Hamiltonian matrix appearing in Eq. (2), rewritten in the current gauge, and $G$ is a diagonal matrix containing the values $G_{*}$ and $G_{\Delta}$ for hub and rim sites, respectively.

The creation and annihilation operators of the quasiparticle eigenstates of this quadratic Hamiltonian will in general be a sum of both particle annihilation and creation operators. These can be obtained through solving the Bogoliubov de-Gennes (BdG) equations

$$
\eta H_{B}(\boldsymbol{k}) \boldsymbol{\phi}_{\boldsymbol{k} \gamma \pm}= \pm E_{\boldsymbol{k} \gamma} \boldsymbol{\phi}_{\boldsymbol{k} \gamma \pm}
$$

where $E_{\boldsymbol{k} \gamma} \geq 0$ and $\eta=\left(\begin{array}{cc}\mathbb{1}_{M \times M} & 0 \\ 0 & -\mathbb{1}_{M \times M}\end{array}\right)$ (see, for instance, 46]). The energies of the Bogoliubov modes are given by $E_{\boldsymbol{k} \gamma}$ where $\gamma$ labels the band index. The quasiparticle operators which diagonalize $\hat{H}_{B}$ are determined from the BdG eigenvectors as $\hat{\alpha}_{\boldsymbol{k} \gamma}=\boldsymbol{\phi}_{\boldsymbol{k} \gamma+}^{\dagger} \eta \boldsymbol{\delta} \hat{\boldsymbol{a}}_{\boldsymbol{k}}$.

The excitation spectrum for a typical parameter set is shown in Fig. 3. It is seen that the interactions give dispersion to the excitation spectrum, which is completely flat at the single-particle level. The excitations about each vortex configuration yield a gapless Goldstone mode due to the broken $\mathrm{U}(1)$ superfluid phase. These have the dispersion $\sim \hbar \sqrt{\left(c_{1} k_{1}\right)^{2}+\left(c_{2} k_{2}\right)^{2}}$ where $k_{1,2}=\boldsymbol{k} \cdot \mathbf{v}_{1,2}$ and $c_{1,2}$ is the speed of sound along the $\mathbf{v}_{1,2}$ lattice vectors.

\section{B. Computation of Degeneracy Lifting}

Having the excitation spectra at hand, we now move on to discuss the resulting degeneracy lifting. We have calculated the thermal and quantum contributions to the free energy in Eq. (15) at a range of values of the input parameters $U_{*} / t, U_{*} / U_{\Delta}, n_{*}$ and, for the thermal part, $T / t$. For each parameter configuration we obtained the band energies by diagonalizing $H_{B}(\boldsymbol{k})$ from Eq. (19) at a uniformly spaced grid of momenta in the Brillouin zone

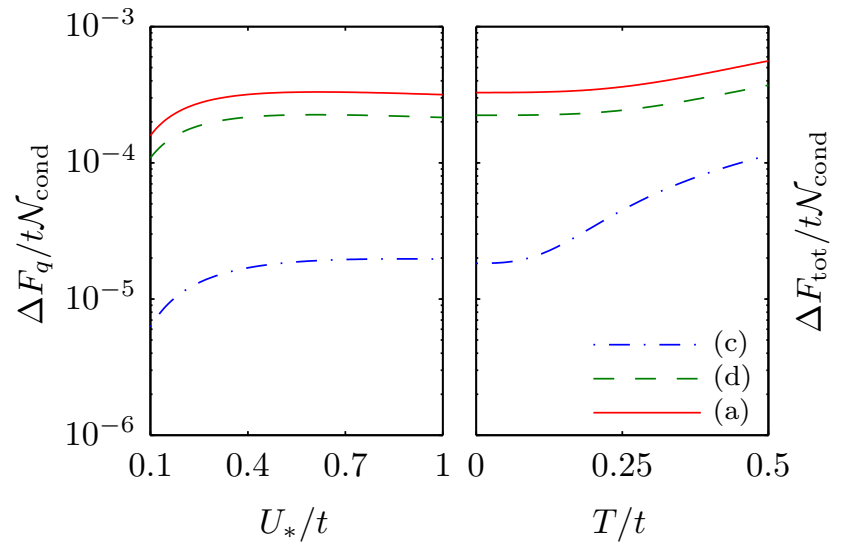

FIG. 4. (Color online) Left: Quantum free energy difference per condensed particle with respect to state (b) from Fig. 2 with $n_{*}=6, U_{*}=U_{\Delta}$. Right: Total free energy difference at the same $n_{*}$ and $U_{*} / U_{\Delta}$, for finite temperature and $U_{*} / t=$ 0.5 .

47. Convergence as a function of the grid spacing was checked for each parameter set.

Results for a range of parameters are shown in Fig. 4. We have plotted the differences of free energies of states (a), (c) and (d) with respect to state (b), $\Delta F_{a, c, d}=$ $F_{a, c, d}-F_{b}$ using the labelling of Fig. 2, As seen in the left-hand side of this figure, the resulting free energy difference is always positive and so state (b) has the lowest free energy. Thermal fluctuations further enhance this degeneracy lifting as shown in the right-hand side of this figure.

In addition to determining the ground state, we also observe that state (c) is universally the highest in free energy. States (a) and (d) are typically ordered as in Fig. 4 but cases were found in which their free energy curves cross. The geometric mean of the sound speeds along the two lattice vectors $\sqrt{c_{1} c_{2}}$ is always lowest for (b) and highest for (c) which explains the ordering of the thermal contribution to the free energy at low temperatures.

\section{The Condensate Depletion}

Having established that state (b) has the lowest overall free energy, we now move on to discuss its stability. For Bogoliubov theory to be valid, one must have that the number of particles excited out of the condensate is small compared to the number of condensed particles. The depletion, like the free energy, can be separated into a quantum and thermal contribution, which we denote by $\mathcal{N}_{\mathrm{q}}$ and $\mathcal{N}_{\text {th }}$, respectively. For the above analysis to be correct we must have $\mathcal{N}_{\text {dep }}=\mathcal{N}_{\mathrm{q}}+\mathcal{N}_{\text {th }} \ll \mathcal{N}_{\text {cond }}$. From the solution of the Bogoliubov-de Gennes equation 20 


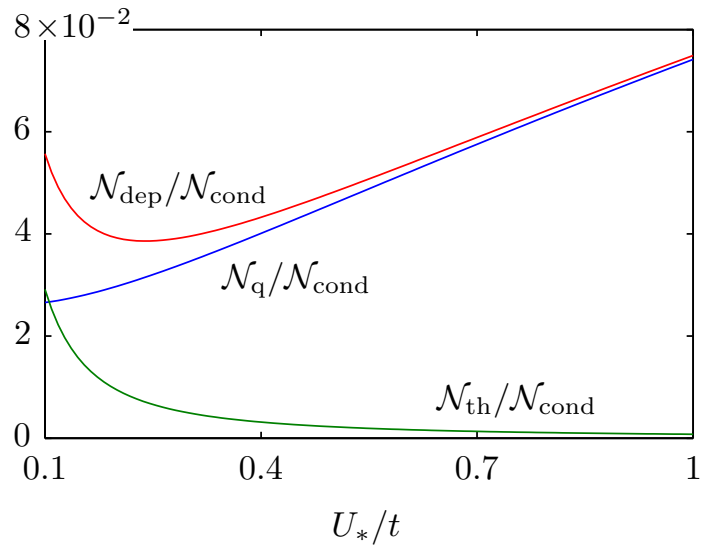

FIG. 5. (Color online) The quantum, thermal and total depletion per condensed particle for a system consisting of $20 \times 20$ unit cells at $n_{*}=6, U_{*}=U_{\Delta}$ and $T=t / 10$.

the depletion can be expressed as

$$
\begin{aligned}
\mathcal{N}_{\mathrm{q}} & =\frac{1}{2} \sum_{\boldsymbol{k} \gamma} \boldsymbol{\phi}_{\boldsymbol{k} \gamma+}^{\dagger}(\mathbb{1}-\eta) \boldsymbol{\phi}_{\boldsymbol{k} \gamma+} \\
\mathcal{N}_{\mathrm{th}} & =\sum_{\boldsymbol{k} \gamma} \boldsymbol{\phi}_{\boldsymbol{k} \gamma+}^{\dagger} \boldsymbol{\phi}_{\boldsymbol{k} \gamma+} f\left(E_{\boldsymbol{k} \gamma}\right)
\end{aligned}
$$

where $f(x)=\left(\mathrm{e}^{\beta x}-1\right)^{-1}$ is the Bose Einstein distribution function.

While the quantum depletion converges, the thermal depletion integral has a logarithmic infrared divergence due to the Goldstone mode. Such divergences are typical for two-dimensional systems 41. Finite size effects will remove this divergence and can be crudely taken into account by using a small-momentum cutoff of $2 \pi / L$ where $L^{2}$ is the system size. Consequently, the thermal depletion will scale as $\ln (L)$ for sufficiently large $L$.

Figure 5 shows the quantum and thermal contributions to the total depletion at experimentally feasible parameters. Quite interestingly, the total depletion exhibits a non-monotonic behavior as a function of the Hubbard interaction parameters. In typical condensed systems, depletion increases monotonically as a function of the interactions 48. A similar minimum was found for all parameter ranges tested. This can be attributed to the flatness of the non-interacting band structure. That is, as interactions are decreased, the Bogoliubov band structure (c.f. Fig. 3) becomes flatter and so thermal excitations are created more easily. When $U_{*}=U_{\Delta}=0$ the Bogoliubov spectrum reduces to completely flat bands and the thermal depletion will diverge. For the chosen parameters in this figure the depletion is always less than $10 \%$. The depletion can be further decreased by choosing larger average density per site.

\section{CONCLUSION}

In this work we have argued that, despite the large degeneracies associated with the non-interacting spectrum of the dice lattice at half-flux per plaquette, fluctuations will select a unique ground state when weak interactions are included. In particular, we have established that quantum and thermal fluctuations select vortex lattice state (b) out of the periodic states shown in Fig. 2 which are degenerate at the level of mean field theory. The stability of the resulting state was established for a range of parameters by analyzing the quantum and thermal depletion.

Although a thorough analysis of the non-periodic states is beyond the scope of the current work, we will briefly comment on them now. As discussed in Sec. III, one can obtain vortex lattices (b,c,d) from state (a) through the insertion of domain walls. At the level of mean field theory, these domain walls cost no energy to create and do not interact. Therefore, through insertion of domain walls one can obtain non-periodic vortex configurations as well as vortex lattices with larger unit cell size, all of which are degenerate at the level of mean field theory with the configurations shown in Fig. 2. On the other hand, including quantum and thermal fluctuations will cause the domain walls to interact. Preliminary calculations of free energy shifts have in fact shown that type II domain walls repel each other, so that the free energy is minimized when they are infinitely separated which is equivalent to no domain walls being present. On the other hand, type I domain walls were found to attract each other, until state (b) is reached. This lends credence to the proposition that state (b) is indeed the state of lowest free energy that is ultimately selected by quantum fluctuations. Considering this, a complete analysis of the fluctuation-mediated interactions between the domain walls and their ensuing dynamics should be an interesting direction for future work.

As has been noted near the end of section III B, macroscopic considerations may also allow for more energetic mean-field domain walls and point defects. Another interesting direction for future research would be to verify whether such defects are indeed stable. Consider again the double asymptotically domain wall-free configuration described at the end of Sec. IIIB. From the viewpoint of the time-dependent Gross-Pitaevskii equation, we can imagine assigning random vorticities and consistent phases, if they can be found, to the plaquettes in the intermediate region and random particle numbers to the sites and then tracking their time evolution. Interestingly, the plaquette vorticities cannot change through smooth time evolution, so we should expect different vorticity distributions to correspond to distinct hydrodynamic solutions. However, there might not always be global steady-state solutions. If, on the other hand, energetic domain walls and point defects are found to be stable, it would enable numerous further investigations to be carried out, such as of the interplay between mas- 
sive and zero-energy domain walls and their free energymediated interactions.

\section{ACKNOWLEDGMENTS}

We would like to thank Dmitry Kovrizhin and Gunnar Möller for fruitful discussions. We are grateful for funding from a Roth Studentship from the Imperial College London Department of Mathematics (MP) and the European Union's Seventh Framework Programme for research, technological development, and demonstration under grant agreement PCIG-GA-2013-631002 (RB).
[1] N. Goldman, G. Juzeliunas, P. Ohberg, and I. B. Spielman, (2013), arXiv:1308.6533

[2] M. Aidelsburger, M. Atala, M. Lohse, J. T. Barreiro, B. Paredes, and I. Bloch, Phys. Rev. Lett. 111, 185301 (2013).

[3] H. Miyake, G. A. Siviloglou, C. J. Kennedy, W. C. Burton, and W. Ketterle, Phys. Rev. Lett. 111, 185302 (2013)

[4] G.-B. Jo, J. Guzman, C. K. Thomas, P. Hosur, A. Vishwanath, and D. M. Stamper-Kurn, Phys. Rev. Lett. 108, $045305(2012)$

[5] N. Cooper, Adv. Phys. 57, 539 (2008)

[6] A. L. Fetter, Rev. Mod. Phys. 81, 647 (2009).

[7] S. Teitel and C. Jayaprakash, Phys. Rev. Lett. 51, 1999 (1983)

[8] T. C. Halsey, Phys. Rev. B 31, 5728 (1985).

[9] J. P. Straley and G. M. Barnett, Phys. Rev. B 48, 3309 (1993)

[10] S. Powell, R. Barnett, R. Sensarma, and S. Das Sarma, Phys. Rev. Lett. 104, 255303 (2010).

[11] J. Vidal, R. Mosseri, and B. Douçot, Phys. Rev. Lett. 81, $5888(1998)$

[12] E. Tang, J.-W. Mei, and X.-G. Wen, Phys. Rev. Lett. 106, $236802(2011)$

[13] K. Sun, Z. Gu, H. Katsura, and S. Das Sarma, Phys. Rev. Lett. 106, 236803 (2011)

[14] T. Neupert, L. Santos, C. Chamon, and C. Mudry, Phys. Rev. Lett. 106, 236804 (2011)

[15] F. Wang and Y. Ran, Phys. Rev. B 84, 241103 (2011).

[16] D. L. Bergman, C. Wu, and L. Balents, Phys. Rev. B 78, $125104(2008)$

[17] S. D. Huber and E. Altman, Phys. Rev. B 82, 184502 (2010)

[18] S. K. Baur and N. R. Cooper, Phys. Rev. Lett. 109, $265301(2012)$

[19] Y.-Z. You, Z. Chen, X.-Q. Sun, and H. Zhai, Phys. Rev. Lett. 109, $265302(2012)$.

[20] X. Li, E. Zhao, and W. V. Liu, Nat. Commun. 4, 1523 (2013)

[21] N. Y. Yao, A. V. Gorshkov, C. R. Laumann, A. M. Läuchli, J. Ye, and M. D. Lukin, Phys. Rev. Lett. 110, $185302(2013)$

[22] S. E. Korshunov, Phys. Rev. B 63, 134503 (2001)

[23] S. E. Korshunov and B. Douçot, Phys. Rev. B 70, 134507 (2004)

[24] S. E. Korshunov, Phys. Rev. B 71, 174501 (2005).

[25] B. Sutherland, Phys. Rev. B 34, 5208 (1986)
[26] T. Morita and T. Horiguchi, J. Math. Phys. 13, 1243 (1972)

[27] V. Cataudella and R. Fazio, EPL (Europhysics Letters) 61, 341 (2003)

[28] M. Rizzi, V. Cataudella, and R. Fazio, Phys. Rev. B 73, $144511(2006)$

[29] D. Bercioux, D. F. Urban, H. Grabert, and W. Häusler, Phys. Rev. A 80, 063603 (2009)

[30] A. A. Burkov and E. Demler, Phys. Rev. Lett. 96, 180406 (2006).

[31] G. Möller and N. R. Cooper, Phys. Rev. Lett. 108, $045306(2012)$

[32] C. C. Abilio, P. Butaud, T. Fournier, B. Pannetier, J. Vidal, S. Tedesco, and B. Dalzotto, Phys. Rev. Lett. 83, $5102(1999)$

[33] E. Serret, P. Butaud, and B. Pannetier, EPL (Europhysics Letters) 59, 225 (2002)

[34] M. Tesei, R. Théron, and P. Martinoli, Physica C: Superconductivity 437438, 328 (2006)

[35] J. Villain, R. Bidaux, J. P. Carton, and R. Conte, Journal de Physique 41, 1263 (1980)

[36] C. L. Henley, Phys. Rev. Lett. 62, 2056 (1989).

[37] J. L. Song, G. W. Semenoff, and F. Zhou, Phys. Rev. Lett. 98, 160408 (2007)

[38] A. M. Turner, R. Barnett, E. Demler, and A. Vishwanath, Phys. Rev. Lett. 98, 190404 (2007)

[39] E. Zhao and W. V. Liu, Phys. Rev. Lett. 100, 160403 (2008)

[40] R. Barnett, S. Powell, T. Graß, M. Lewenstein, and S. Das Sarma, Phys. Rev. A 85, 023615 (2012)

[41] N. D. Mermin and H. Wagner, Phys. Rev. Lett. 17, 1133 (1966).

[42] P. W. Anderson, Phys. Rev. 109, 1492 (1958).

[43] With the exception of triangular clusters of like-vortices around rim sites which could be regarded as cyclic chains of length 3. These cannot occur.

[44] We can in fact only insert one of the type I domain walls shown and all walls parallel to it, but we cannot insert any two type I domain walls at an angle.

[45] Further details of the phase permutations comprising each type of domain wall and figures of single domain walls inserted into state (a) are given in Ref. 22.

[46] J.-P. Blaizot and G. Ripka, Quantum theory of finite systems (MIT press Cambridge, 1986).

[47] H. J. Monkhorst and J. D. Pack, Phys. Rev. B 13, 5188 (1976)

[48] C. Pethick and H. Smith, Bose-Einstein Condensation in Dilute Gases, 2nd ed. (Cambridge University Press, 2008). 